\title{
Determinación de las causas de reproceso durante la fabricación de radiadores de cobre- latón y propuesta de acciones correctivas
}

\author{
S.M. Cerrud-Sánchez, A. Ortiz-Prado, R. Schouwenaars y V.H. Jacobo-Armendáriz \\ Departamento de Ingeniería Mecánica \\ Facultad de Ingeniería, UNAM \\ E-mail:armandoo@servidor.unam.m
}

(recibido: enero de 2002; aceptado: noviembre 2002)

Resumen

La fabricación de radiadores cobre-latón para uso automotriz se realiza tanto con métodos manuales como con sistemas automatizados, o bien, semiautomatizados. Por contar con un mejor control sobre las variables críticas, los sistemas automatizados generan una mayor calidad y eficiencia del producto que los manuales; sin embargo, cuando se tiene una gran diversidad de modelos de radiador y en ocasiones es necesario producir lotes pequeños, resulta más conveniente el uso de métodos manuales y sólo automatizar algunas operaciones. En empresas que involucran una gran variedad de productos y bajos volúmenes de producción, se presentan una serie de problemas en aspectos de calidad y excesos de retrabajo, así como en desperdicio de soldadura, lo cual tiene repercusiones inherentes en términos de costos de producción. En este artículo se presenta el método para identificar en cada una de las etapas del proceso de fabricación de radiadores, las causas que generan los efectos mencionados, así como las estrategias establecidas para mejorar la calidad y productividad de la empresa bajo estudio. A pesar de que el método está orientado hacia una empresa en particular, la problemática es representativa de una gran parte de la industria nacional.

Descriptores: calidad, productividad, análisis de falla, radiadores automotrices.

\section{Abstract}

The manufacturing of automotive radiators of copper-brass alloys requires a series of very complex operations. For this reason the automation is complicate and requires the use of intensive handwork. In automated systems, better control of the critical variables is the main reason of the higher quality products. However, when a great diversity of models and small production volumes are required, it is more convenient to use manual operations. In such enterprises, a lot of problems are easily detected, such as quality problems, excess rework and waste of soldering material, accompanied by their repercussions on production costs. This paper presents the method used to identify the causes of the problems and the strategies to solve them in each manufacturing step. This paper presents the specific case of one specific industry, but reflects a lot of the problems of the mexican industry in similar enterprises in Mexico. 


\section{Introducción}

Los radiadores cobre-latón para uso automotriz se han empleado prácticamente desde el inicio de los motores de combustión interna, esto se ha traducido en una amplia experiencia en su proceso de producción. Por sus características, el número de operaciones requerido para su fabricación es elevado (el número de piezas que los constituyen generalmente supera a las 50), razón por la cual es necesaria una gran cantidad de operaciones, varias de las cuales son difíciles de automatizar.

Aún cuando este tipo de radiadores presenta notables ventajas en su operación, han sido sustituidos de 20 años a la fecha por los de aluminio-polímeros reforzados en equipo original, esto por la necesidad del diseñador automotriz de reducir el peso del vehículo a la vez de permitir una mayor automatización en la fabricación del radiador. Sin embargo, las ventajas del radiador cobre-latón (mayor eficiencia en la transferencia de calor, duración, facilidad de mantenimiento) han provocado que permanezca en el mercado de refacciones, no observándose en el panorama actual una condición que permita prever su desaparición.

La mayor cantidad de operaciones que involucra la fabricación del radiador cobre-latón, así como la comple- jidad de éstas, dan lugar a un uso intensivo de mano de obra. Los costos adicionales que involucran las operaciones de retrabajo y los desperdicios de material que se presentan, son algunos de los retos necesarios para conquistar y garantizar la competitividad del producto, y por ende, su permanencia en el mercado. Para facilitar la compresión del proceso de producción, se presenta en la figura 1 el diagrama del proceso de manufactura correspondiente a la empresa bajo estudio.

Considerando lo anterior, se planteó el desarrollo de un proyecto con la orientación inicial de determinar las causas que provocan fugas y retrabajos en etapas críticas del proceso.

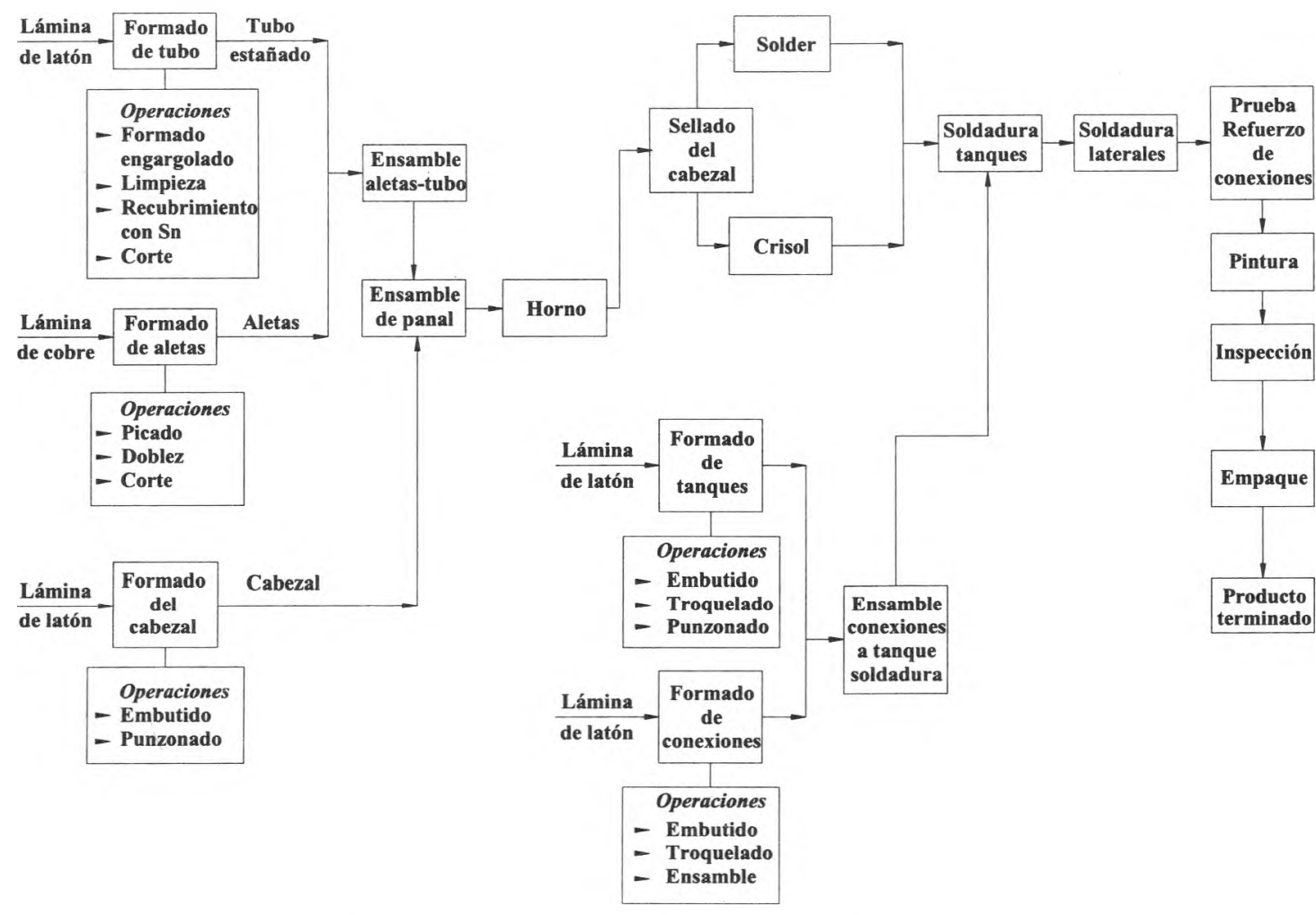

Figura 1. Diagrama general de proceso de radiador 
No obstante, después de dar seguimiento a las diversas operaciones, se observó que en el subconjunto denominado panal con tanque, el número de fallas era aún superior que en el radiador completo, concluyéndose que los inconvenientes no necesariamente se generan durante la última etapa, en la cual se llevan a efecto operaciones de soldadura (ensamble de soportes), idea que en un principio era la hipótesis. Por tal motivo, fue necesario determinar el efecto que cada una de las etapas de fabricación tiene sobre las fallas observadas, primero se procedió a estructurar una revisión integral del proceso con la finalidad de identificar en cada una de las fases las causas de problemas relacionados con los siguientes factores: materiales, mano de obra, métodos, maquinaria, medio ambiente y mediciones. Con base en los resultados obtenidos a partir del análisis causa-efecto, se establecieron estrategias y recomendaciones con el objeto de realizar una mejor práctica y reducir los costos de producción, a la vez de aumentar la productividad de la planta.

\section{Descripción de la metodología}

Se identificaron los modelos de radiador, que desde el punto de vista del retrabajo requerido representaban mayor problema. Para el presente estudio se seleccionaron 7 diferentes tipos de radiador. Posteriormente, se procedió al seguimiento de lotes de radiadores durante su producción, de donde se obtuvieron muestras para su inspección detallada en el laboratorio.

La visión integral del problema representó el análisis de las diferentes piezas que constituyen el radiador (Figura 2), sus procesos de producción y los métodos de ensamble. Para ello, se llevaron a efecto los siguientes estudios:

a. Medición de las ranuras del cabezal, tanto en ancho como en longitud (dos cabezales por tipo).

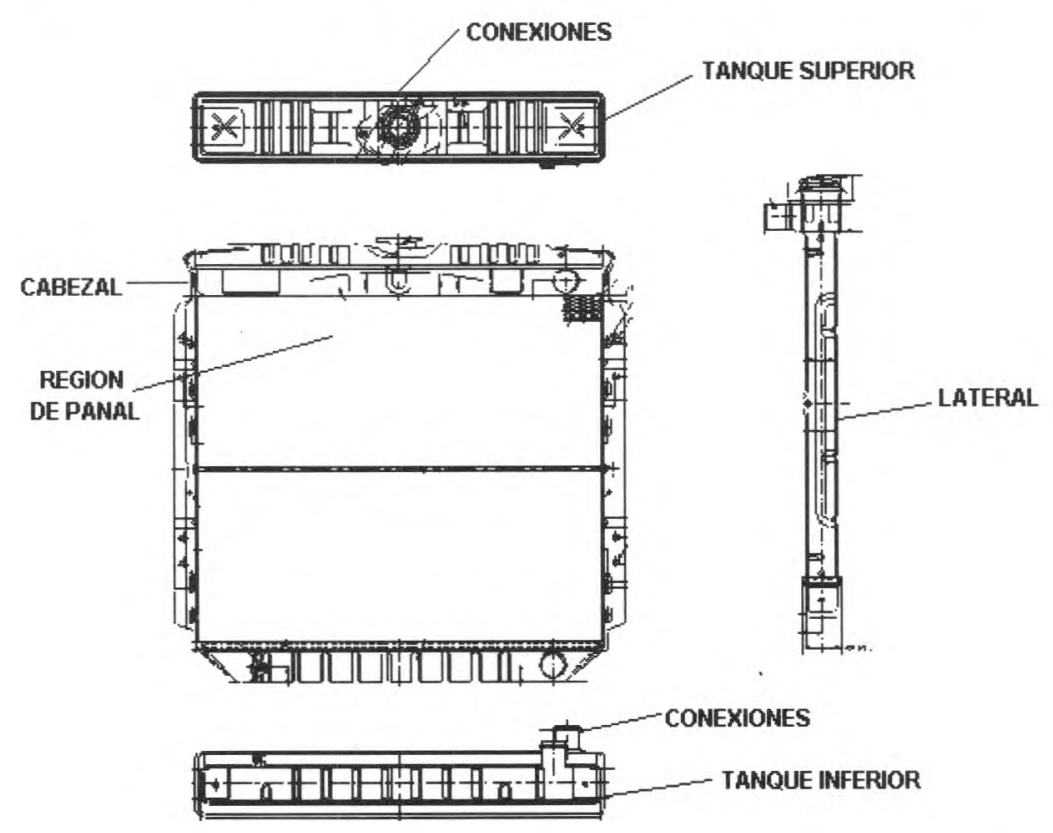

VISTA SUPERIOR DE CABEZAL

Figura 2. Componentes de un radiador típico 
b. Análisis estadístico de los datos referentes a las dimensiones de las ranuras del cabezal.

c. Inspección a bajos aumentos del estado de las ranuras del cabezal.

d. Análisis geométrico y dimensional de los tubos, además de las condiciones de su engargolado, tanto por la parte exterior, como por la parte interior de los mismos.

e. Análisis de las zonas en donde se detectaron fugas con la finalidad de determinar sus causas.

f. Análisis de las condiciones presentadas por el cabezal ya soldado.

g. Comparación estadística de las dimensiones de las ranuras de cabezal con las de sus respectivos tubos.

h. Análisis de la incidencia de fallas que se presentan durante el proceso de producción.

i. Análisis de las holguras entre tubo y cabezal en piezas soldadas.

j. Inspección mediante microscopia electrónica de barrido de las zonas en donde se detectaron inconvenientes.

Considerando el anterior procedimiento fue posible determinar las condiciones que permiten alcanzar las siguientes metas:

1. Determinar, con base en la estadística y el seguimiento de las piezas, las estaciones que representan problemas de calidad.

2. Determinar las causas que provocan las fallas de hermeticidad.

3. Definir acciones correctivas en los procesos que sean detectados como conflictivos desde el punto de vista del estudio.

4. Validar el efecto de las acciones correctivas y su trascendencia.

Por último y con la finalidad de identificar las causas que generan defectos en las diversas etapas del proceso de producción del radiador, se realizó una tormenta de ideas para la elaboración del diagrama causa-efecto correspondiente. Los resultados que surgieron mediante estas técnicas para el análisis de problemas son presentados en la figura 3.

\section{Resultados}

Con la finalidad de lograr una mayor claridad en la presentación y análisis del estudio durante el proceso de producción del radiador y la inspección en laboratorio, se presentan los resultados, considerando el orden en el que se realiza el proceso de fabricación.

\section{Formado de tubo}

La geometría del tubo no corresponde con la definida en los procedimientos de control de calidad de la empresa, en la mayoría de los casos estudiados, existe una tendencia a que las dimensiones no cumplan con las especificaciones, de acuerdo con el análisis estadístico de los datos generados. En la figura 4 se presentan los resultados de la medición de un tubo de $5 / 8$ ", en donde existen mediciones fuera de los límites, o bien, muy cercanas a ellos, lo que trae consecuencias en la relación de ensamble con las ranuras y el consiguiente inconveniente en el proceso de soldadura.

\section{Formado de aletas}

No se encontró inconveniente o efecto colateral que pudiera influir negativamente en alguna etapa posterior del proceso.

\section{Formado del cabezal}

El problema más conflictivo que se deriva de esta pieza, se debe a las condiciones de punzonado, ya que el elevado número de punzones (en ocasiones superior a 200) y las características de la propia operación, dan como resultado ranuras fuera de especificación y una importante dispersión en las dimensiones (Tabla 1, figura 5), que aunada con la dispersión asociada a los tubos, da lugar a excesos de holgura (como se muestra en las gráficas gaussianas para ranuras y tubos en la figura 6) o a la deformación de los propios tubos durante el ensamble. En el perfil de los cabezales existen diferencias (geométricas y dimensionales) que se traducen en inconvenientes durante el ensamble con los tanques. 


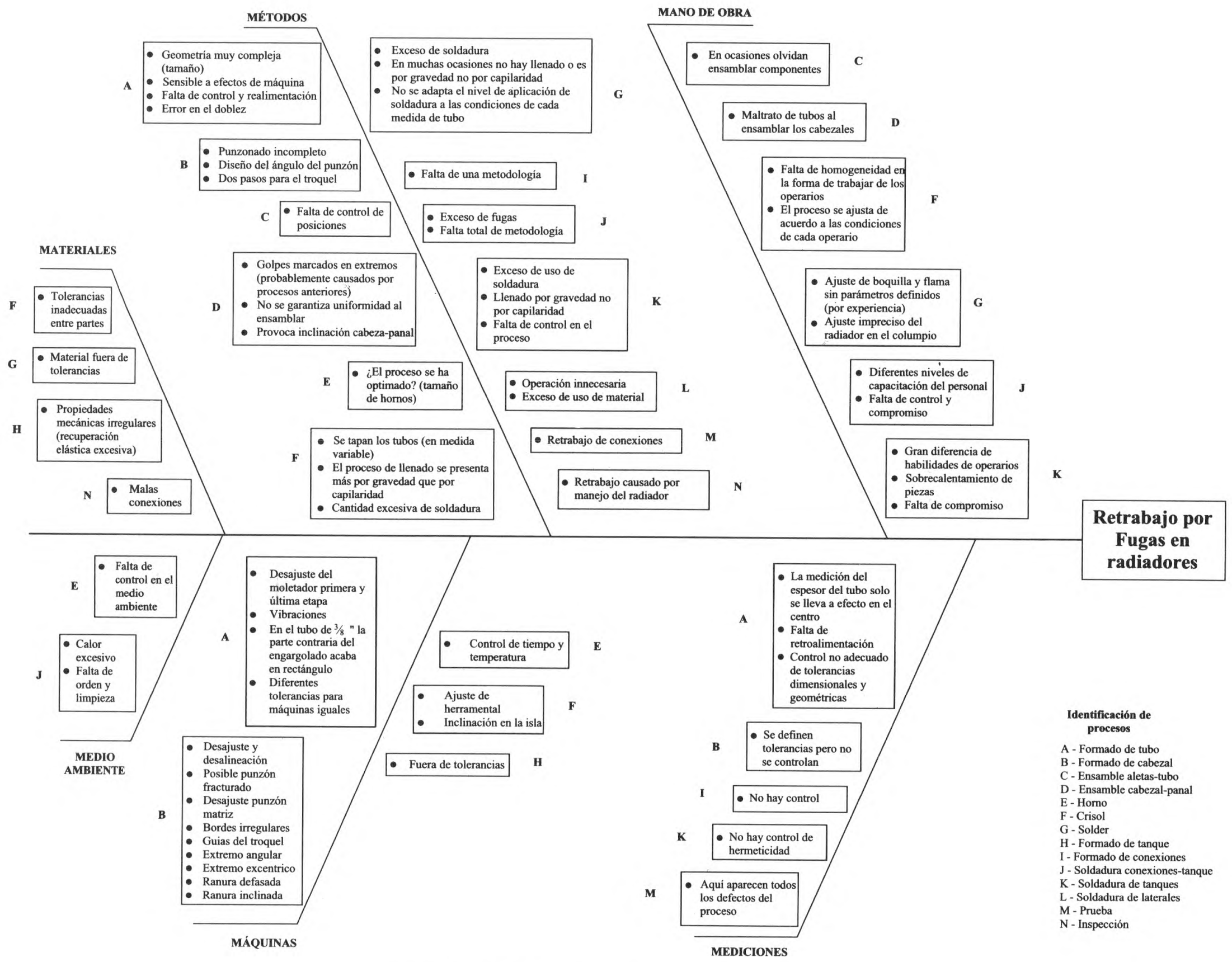


DOI: http://dx.doi.org/10.22201/fi.25940732e.2003.04n3.011

Determinación de las causas de reproceso durante la fabricación de radiadores ...

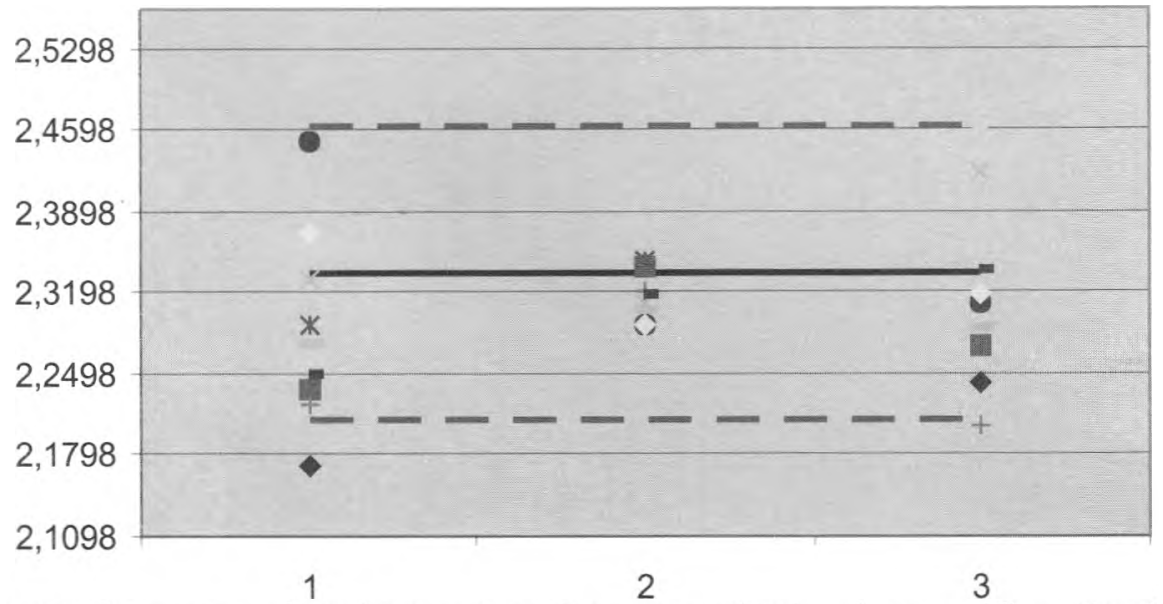

Figura 4. Mediciones de espesor de tubo por posición contra límite de especificación. La linea continua representa el valor nominal, mientras que las punteadas los limites de especificación. Los símbolos representan cada una de las mediciones realizadas, por su parte el 1 y 3 representan medición en el extremo y 2 medición en el centro

\section{Ancho}

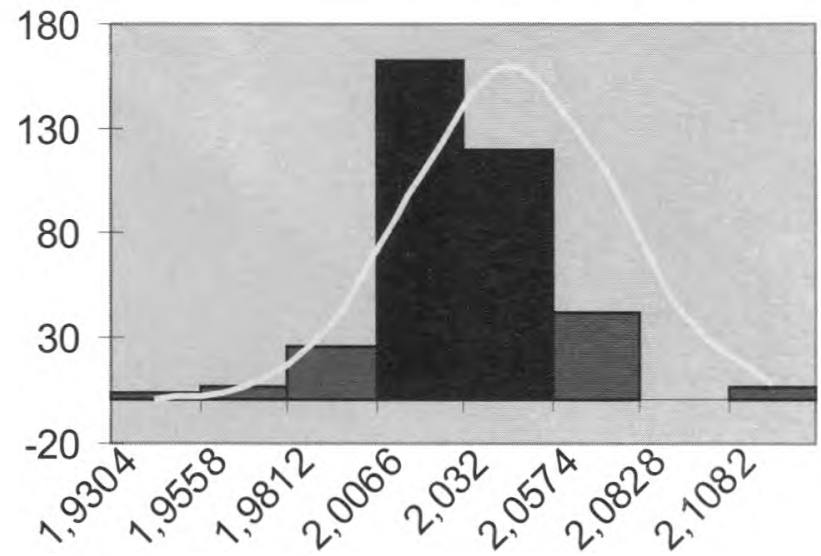

aceptado $\square$ rechazado gaussiano

Figura 5. Histograma de frecuencias del modelo de radiador de la tabla 1

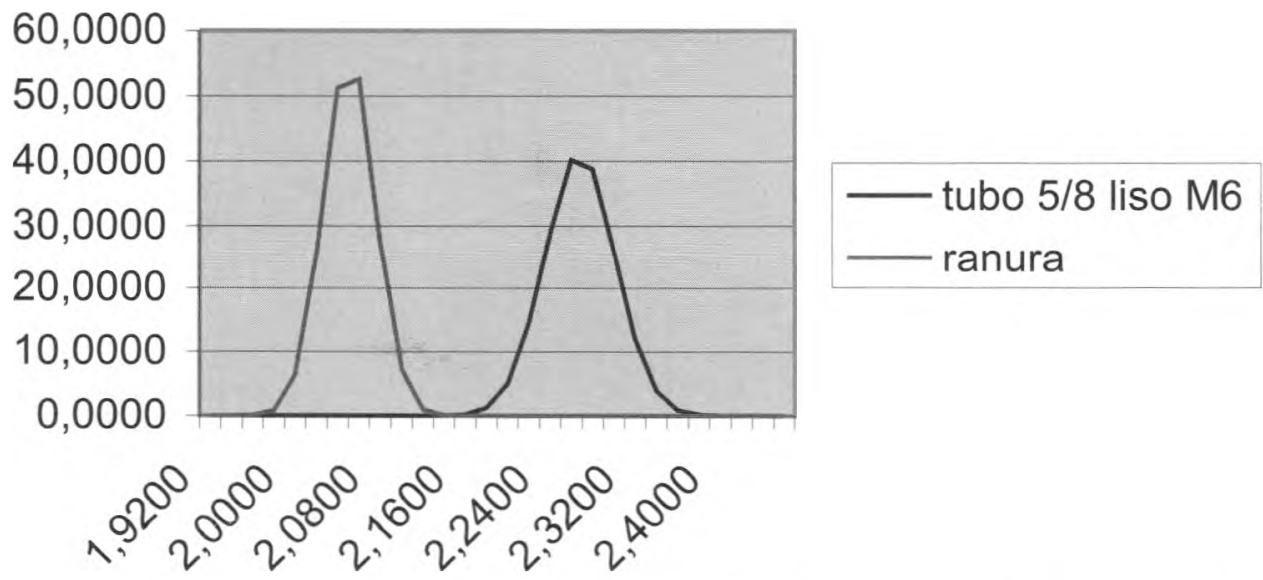

Figura 6. Gráficas de dimensiones de anchos y espesores para ranuras y tubos. Existe una holgura excesiva para la relación de ensamble por un lado y para la aplicación de la soldadura por el otro 
DOI: http://dx.doi.org/10.22201/fi.25940732e.2003.04n3.011

S.M. Cerrud-Sánchez, A. Ortiz-Prado, R. Schouwenaars, V.H. Jacobo-Armendáriz

Tabla 1. Datos estadísticos referentes al ancho de la ranura en el cabezal de un modelo de radiador representativo

\begin{tabular}{llll}
\hline Mínimo & 1.9304 & Tolerancia & $2.03 \pm 0.03$ \\
Máximo & 2.1082 & Promedio & 2.03350042 \\
& Desv. Est. & 0.03020606 \\
Bajo & 36. & 7.6 \\
Aceptado & 390. & 82.3 \\
Alto & 48. & 10.1 \\
& 474. & \\
\hline
\end{tabular}

\section{Ensamble de panal (aletas-tubos)}

No se encontró inconveniente o efecto colateral que pudiera influir negativamente en alguna etapa posterior del proceso.

Ensamble de cabezal a panal

El proceso es heterogéneo, dando lugar a maltrato (deformación) de los extremos de los tubos, así como del propio cabezal.

\section{Horno}

En esta etapa se generan inconvenientes al no existir un control óptimo de las variables, lo que se traduce en falta de fijación (fusión) entre las piezas (aletas y tubos) lo que provoca la necesidad de que el panal sea enviado de nueva cuenta al horno con las repercusiones en el costo y tiempo de proceso.

\section{Soldadura de cabezal}

El proceso de soldadura se realiza por dos métodos, semiautomático y crisol.

\section{Proceso semiautomático}

Se trata de un proceso en donde el panal con cabezal pasa subsecuentemente por una zona de calentamiento por flama, suministro de soldadura en estado líquido, enfriamiento e inyección de aire a presión por el interior de los tubos; esto con la finalidad de eliminar cualquier acumulación de soldadura que tienda a taponar éstos. En general, en este proceso se produce un menor número de fallas por hermeticidad que en Crisol. Sin embargo, se observa que la cantidad de soldadura es excesiva, lo que se traduce en gastos innecesarios, así como en pérdidas de eficiencia térmica del radiador, debida al taponado parcial o total de algunos tubos. La forma de calentamiento y las holguras entre tubo y cabezal provocan que el llenado sea por gravedad y no por capilaridad, que es lo deseable.

\section{Crisol}

En este proceso se sumerge parcialmente el cabezal en un recipiente con soldadura en estado líquido. Por sus características, este método es dependiente de la habilidad y prácticas del operador. El ajuste en la inclinación de la isla (recipiente con metal líquido) es uno de los parámetros que producen un flujo diferencial. Asimismo, de la observación de las prácticas de los operadores se constata que el llenado es por gravedad, ya que se produce por recarga del nivel de la isla. La variación en los procedimientos es una de las principales causas de la heterogeneidad del producto y se traduce también en la frecuencia de defectos (falta de hermeticidad entre tubo y cabezal). Este procedimiento es todavía más crítico que en el proceso semiautomático en términos de desperdicio de soldadura. En la figura 7 se presenta un error común en la aplicación de la soldadura por el método bajo análisis.

En estos métodos de unión (semiautomático y crisol) se generan una gran cantidad de inconvenientes, en la figura 8 se muestra la clasificación de las deficiencias encontradas en un radiador típico.

\section{Formado de tanques}

La operación empleada para producir los tanques (estampado y punzonado), es dependiente de las condiciones de las herramientas, así como de las características del material. Los inconvenientes que se presentan durante la soldadura de tanques se pueden relacionar con la 
habilidad y prácticas de los soldadores, así como con las características dimensionales inadecuadas entre tanque $y$ cabezal (holgura excesiva o interferencias).

Independientemente del desajuste de las herramientas, un problema fundamental se relaciona con la recuperación elástica del material después de su formado (embutido), así como con defectos en el troquelado (arranques y desgarres), lo cual genera problemas en la soldadura.

\section{Soldado de conexiones}

El personal con menor experiencia y habilidad es el que realiza esta operación, ya que aquí existe una gran dispersión en el nivel de capacitación y compromiso. Todo esto trae como consecuencia que en estaciones posteriores se vuelva a aplicar soldadura para garantizar una adecuada unión de la conexión. En este caso, el retrabajo se ha convertido en una etapa más del proceso de producción.

\section{Soldadura de tanques}

Al inicio del análisis se consideró que esta era la etapa más difícil del proceso, planteándose la hipótesis de que el calentamiento generaba refusión, y por ende, fugas que eran detectadas y corregidas ya en la etapa de prueba. Sin embargo, de lo observado se concluye que aunque existen notables diferencias en la habilidad y en el compromiso de los operarios, la mayoría de las deficiencias se deben a los inconvenientes generados en etapas anteriores del proceso.

\section{Soldadura de laterales}

En ocasiones, el exceso de calentamiento durante la etapa provoca nuevas fugas; sin embargo, esto no representa la condición normal. Por otra parte, se determinó que se deposita material en exceso y que buena parte de la operación se podría obviar con el consiguiente ahorro de material y mano de obra.

\section{Prueba}

De acuerdo a los procedimientos actuales, esta operación es la responsable de corregir todos los inconvenientes que se han generado a través del proceso.

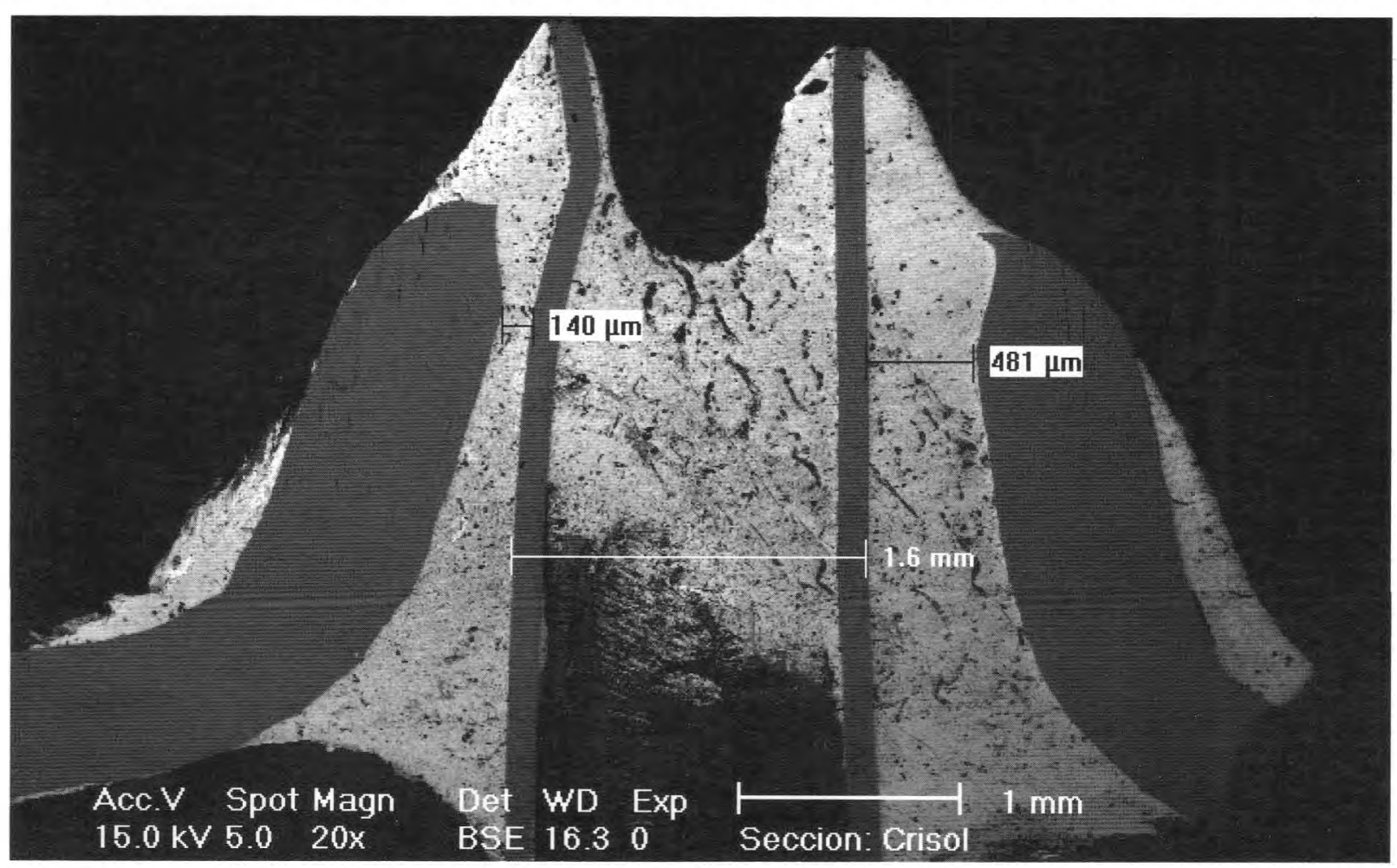

Figura 7. Proceso de soldadura Crisol. Zona cabezal con tubo 20x. Se observa que el tubo está relleno de soldadura y no conserva la misma distancia entre el cabezal de ambos lados 


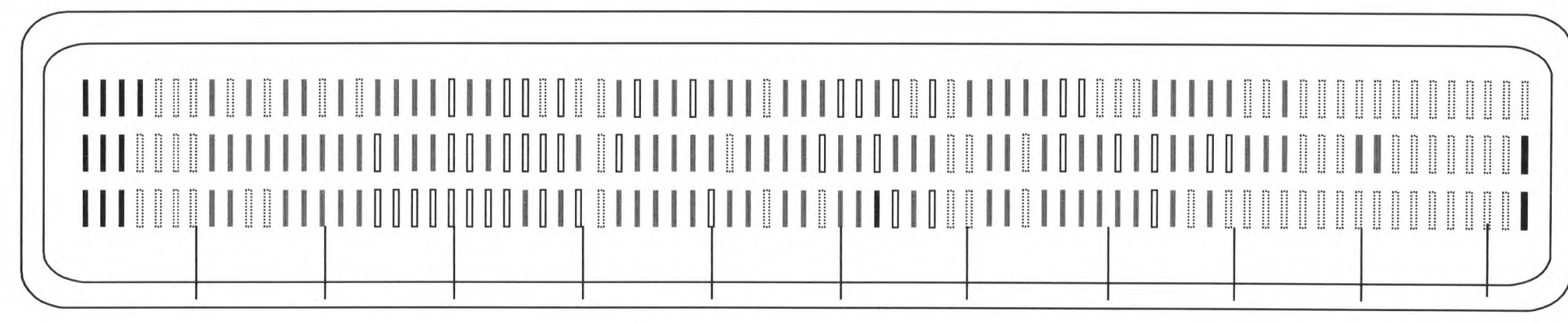

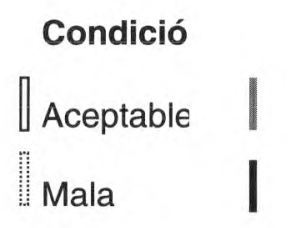

CARACTERIZACIONES DE TUBOS:

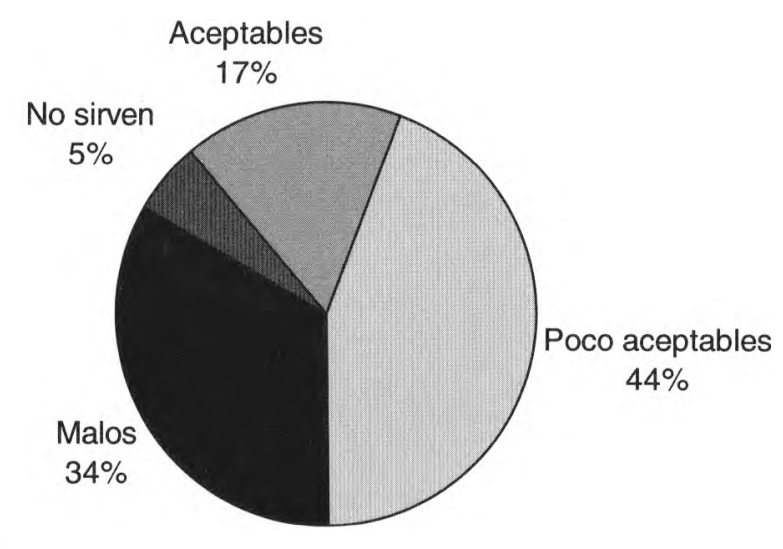

\begin{tabular}{|l|r|}
\hline Aceptables & 40 \\
\hline Poco aceptables & 104 \\
\hline Malos & 80 \\
\hline No sirven & 13 \\
\hline TOTAL & 237 \\
\hline
\end{tabular}

Aceptable: Tubo en buen estado, no tapado o deformado con cantidad de soldadura adecuada.

Poco aceptable: Tubo no tapado, ligeramente deformado y/o con ligero exceso de soldadura o rebaba.

Malos: Tubo deformado y/o con exceso de soldadura o rebaba, pueden presentar taponamiento que puede ir de $1 / 4$ a $3 / 4$ del orificio por soldadura o deformación.

No sirven: Tubos completamente tapados por deformación o soldadura, o bien presentan fuga. 


\section{Pintura}

No se encontró inconveniente o efecto colateral que pudiera generar problemas al producto.

\section{Inspección}

Como la idea es garantizar la calidad al $100 \%$, los operarios tienen que corregir algunos aspectos como el doblado de aletas, que no debieran de existir, pero que se presentan por la falta de cuidado durante la manipulación de los radiadores en todo el proceso.

\section{Transporte y almacenaje entre estaciones}

A pesar de que ésta no es una operación de producción en sentido estricto, sí influye en los costos de producción, ya que existe maltrato causado por la falta de conciencia de los operarios

\section{Discusión y recomendaciones}

A través de este trabajo se comprueba la importancia que la aplicación de diversas técnicas de análisis de la ingeniería industrial tiene en la determinación de las causas de falla en un proceso. Se muestran datos a partir de los cuales es posible definir las acciones correctivas que permitan reducir sensiblemente la frecuencia con la que se presentan fallas. Al inicio fue necesario comprobar la hipótesis plan- teada por el personal técnico de la empresa, el cual consideraba que los problemas se generaban durante la soldadura del tanque y de los laterales.

Los datos que se desprenden del presente estudio, indican que la problemática se produce generalmente en las etapas anteriores de la manufactura del radiador. La investigación realizada permite comprobar el efecto que cada etapa tiene en el retrabajo final del producto. Con esto se descartó la hipótesis original en relación a que la última etapa de producción era la causante de los retrabajos, planteando una tesis en el sentido de que cada una de las etapas de manufactura tiene una aportación en los inconvenientes detectados en el producto terminado.

Por los resultados se concluye también que aún cuando se cuenta con todos los procedimientos de evaluación de materias primas, muchos de éstos no son adecuados para definir aquellas propiedades que representan mayor interés de acuerdo a las necesidades de la planta. En lo que se refiere a los métodos establecidos por el departamento de manufactura, algunos deberán ser actualizados tomando en general las providencias necesarias para su cumplimiento, de tal forma que no queden sólo en papel.

La cantidad de defectos que se presentan en productos que provienen del turno nocturno, permite suponer que las prácticas se relajan aún más en éste.
Esto conlleva a la necesidad de un estudio a detalle con relación a las condiciones de operación y la efectividad de la supervisión en dicho turno.

El proceso de formado del tubo mediante rodillos es muy sensible a los ajustes de las máquinas, considerando además las dimensiones características a los mismos.

Los materiales empleados, en particular la lámina de cobre y de latón, no son evaluados adecuadamente en sus propiedades mecánicas durante su recepción, de tal forma que se debe garantizar la presencia de propiedades homogéneas y adecuadas para su uso.

Se pudo observar que aunque se cuenta con procedimientos documentados, la cultura de la calidad no es un concepto que se encuentre permeado en la planta, de hecho, el enfoque de calidad tiende más a "controlar la calidad" que a producirla en cada etapa del proceso, ésta es la razón fundamental con la que se identifica el alto nivel de retrabajo, que en general, es necesario.

Con base en los inconvenientes observados, se definieron diversas acciones a desarrollar con la finalidad de mejorar las actuales condiciones de producción; la aplicación de cada una de ellas se deberá realizar con base en un criterio de eficiencia que garantice las máximas mejoras del sistema con el mínimo esfuerzo involucrado. 


\section{Problemas y soluciones propuestas}

\section{a. Mejorar la calidad del tubo}

Principalmente en lo que se refiere a su perfil. De los resultados de los estudios de depósitos se desprende la necesidad de modificar los fundentes o productos de limpieza utilizados, previo al estañado del tubo, de tal forma que se elimine de manera radical el empleo de compuestos de bromo por los efectos negativos que éstos tienen sobre la salud. Una opción es emplear tubo electrosoldado en vez del formado por rodillos; sin embargo, cualquier discusión adicional deberá relacionarse con la factibilidad económica de esta sustitución. Para el tubo formado con rodillos se deberá cumplir con los requerimientos de calidad establecidos por la empresa, y no dejar pasar sin corrección los que están fuera de especificación.

\section{b. Cabezal}

En esta pieza se encontraron defectos en la calidad del punzonado, perfil de la pieza (pandeo) y geometría de borde; asimismo, existen desalineamientos en los orificios producidos por el punzonado. Por lo tanto, es necesario cumplir estrictamente con las normas de calidad establecidas, por lo que se deberá controlar el ajuste y estado del herramental de punzonado y embutido, a la vez de las propiedades de la materia prima involucrada (lámina de latón). En este aspecto es fundamental el control de las propiedades mecánicas, así como del espesor de los materiales a través de métodos modernos y normalizados internacionalmente.

\section{c. Ensamble de cabezal con panal}

Se recomienda automatizar la operación de tal forma que se elimine la distorsión generada por la práctica actual de los operadores, ésta produce maltrato sobre cabezal y extremos de tubos. Se considera que el costo de diseñar y construir una prensa con accionamiento neumático para realizar la operación, se justifica con los beneficios que se obtendrán al mejorar la calidad del producto ensamblado. Para garantizar el éxito de la automatización, es necesario primero asegurar que las dimensiones y tolerancias de los cabezales y de los tubos sean congruentes en su relación de ensamble para evitar interferencias u holguras excesivas. En reserva de que se analice y se proceda a la automatización del proceso, es necesario reeducar a los operarios para que se concienticen de la importancia de su trabajo, de tal forma que el ensamble se realice sin causar deterioro a las piezas.

\section{d. Soldadura de cabezal a panal}

El procedimiento semiautomático de soldadura (solder), es difícil que sea empleado en cabezales de 4 hileras de ranuras, pero aquí se presenta como la opción más adecuada para el proceso. Lo anterior, con base en los resultados estadísticos de las fallas de hermeticidad presentadas por el producto, así como en los tiempos de proceso, los cuales son favorables con relación al método de crisol. Por tanto, se recomienda que crisol sólo se emplee para cabezales con 4 hileras o para dimensiones que imposibiliten el uso del proceso semiautomatizado. Para optimar este proceso se recomienda establecer con precisión las condiciones de flama y gasto de material de aporte, de acuerdo a las necesidades de cada modelo. Para crisol es posible automatizar el procedimiento reduciendo los costos y mejorando la calidad del producto.

\section{e. Soldadura de conexiones}

Es necesario incrementary homogeneizar los niveles de capacidad de los operarios. Se tiene que fijar como objetivo la búsqueda y eliminación de los defectos, de tal manera que en las etapas posteriores se elimine el retrabajo que se realiza en conexiones. Es conveniente llevar a cabo un programa de capacitación continuo tendiente a elevar los niveles de habilidad para que de manera gradual se disminuya la cantidad de defectos generados en el proceso, asimismo, es necesario realizar evaluaciones permanentes del proceso para identificar el avance en la mejora de la calidad de soldado de conexiones. Para garantizar el 
éxito de las acciones establecidas es necesario definir y dar cabal cumplimiento a las especificaciones de calidad de soldadura de las conexiones.

\section{f. Refuerzo de laterales}

Como tal, es una operación innecesaria, que se traduce en un mayor consumo de soldadura y en un gasto adicional por mano de obra. Es indispensable para cada situación, analizar la pertinencia de esta etapa y, en su caso, eliminarla.

\section{g. Soldadura tanques}

Esta operación es factible de automatizar, pero para lograrla, es necesario eliminar los inconvenientes relacionados con las tolerancias y perfiles de cabezal y tanque.

h. Manejo y almacenaje de productos en proceso

Con relativa frecuencia se observan daños en las aletas, producto de la manipulación en las diversas etapas. Se desprende entonces la conveniencia de concientizar al personal de la importancia de cuidar al producto durante su manipulación y desde luego su almacenaje.

\section{i. Actitud de los actores} involucrados en las diferentes etapas de producción

Generalmente la actitud que se observa es la de controlar la calidad, no de producirla, esta es una visión, que si bien, fue la común hasta los años 80 's, ha sido ya superada con beneficios tanto en calidad como en costos de producción, ya que se debe generar la calidad más que controlarla.

j. Procedimiento de asignación de incentivos

De acuerdo con lo anterior, los incentivos para los operarios se definen bajo el concepto de productividad, entendiéndose como la cantidad de producto generado por el operario, sin tomar en cuenta la calidad, lo que resulta en una práctica inadecuada, debido a que el operador se ocupa simplemente de producir más sin importarle que esté bien o mal, generando defectos que repercutirán negativamente en la cantidad de mano de obra necesaria por pieza y en consecuencia, en los costos de producción. Por ello, es conveniente plantear un nuevo sistema que incentive la productividad con calidad.

\section{Conclusiones}

Los procesos que emplea la empresa se han traducido, en muchos casos, en una falta de conciencia de la importancia de cada operación sobre la calidad, así como incrementos en el costo final de manufactura del radiador. Esto ha dado como resultado que los responsables de las diferentes etapas se orienten exclusivamente a cuestiones de cantidad y no de calidad. El punto crítico de las empresas es que el retrabajo se da como procedimiento y no como una condición de excepción.

Considerando la situación actual es factible con pequeños esfuerzos mejorar de manera sensible las condiciones de operación de la empresa, de tal forma que se reduzcan las prácticas de retrabajo, los tiempos de producción, el desperdicio de materias primas y el número de horas de mano de obra por pieza producida, dando como resultado la disminución de los costos de producción y una mejora sensible en la calidad del producto. Por lo anterior, se dio inicio a una serie de acciones encaminadas a:

a) Modificar la cultura de la calidad, de tal forma que todos los actores de la producción se involucren en ella.

b) Eliminar o disminuir operaciones que no añaden valor al producto (refuerzo de laterales).

c) Automatizar o semiautomatizar operaciones que a la fecha dan lugar a inconvenientes por la variabilidad de la capacidad de la mano de obra involucrada (soldadura de panal a cabezal, ensamble de panal a cabezal).

d) Diseñar métodos de evaluación del producto en sus diferentes etapas que garanticen la calidad de los subconjuntos producidos.

e) diseñar métodos de recompensa que promuevan la productividad con calidad.

f) Modificar el diseño de algunas de las piezas que 
integran los radiadores, a modo de que se facilite su proceso de automatización (conexiones).

g) Modificar las condiciones de operación de algunas de las etapas (soldadura de conexiones) para que el ambiente de trabajo permita un mejor desempeño de los operadores al disminuir la temperatura y la cantidad de vapores en el ambiente.

Por último, es conveniente mencionar la importancia de una permanencia en el mercado de este tipo de empresas, las cuales hacen uso intensivo de mano de obra, proporcionando los empleos que tanto demanda la sociedad. Por el tipo de producto, el cual se prevé siga siendo requerido, las empresas sólo necesitan que se les brinde la asesoría y el soporte tecnológico para mantener su competitividad a nivel internacional.

\section{Agradecimientos}

Se agradece la participación de los ingenieros Roberto Cisneros, Efraín Ramos e Ignacio Cueva, así como al técnico Germán Alvarez, quienes participaron en las pruebas de laboratorio y aportaron ideas para el desarrollo del diagrama causa-efecto.

\section{Bibliografía sugerida}

Ammerman M. (1997). The Root Cause Analysis. Ed. Kraus Organisation Limited, EUA.

Colin J. (1982). Capilarity Joining. Ed. Jon Wiley \& Sons, EUA.

Curfman L. (1976). Automotive Radiator Construction and Restauration for Antique and Classic. Ed. The Post Group, EUA.

Easterling K. (1993). Introduction to the Physical Metallurgy of Welding. Ed.Butter WorthHeinemann Ltd.
Evans R.(2001). The Managing and Control of Quality. Ed. South Western Thompson learning, EUA.

Feigenbaum A. (1997). Control total de la calidad. Ed. CECSA, México.

Griffith G.K. (1997). Control de calidad. Ed. Prentice Hall, EUA.

Laboucheix V.(1997). Tratado de la calidad total. Ed. Limusa, México.

Lancaster J. F.(1984). Metallurgy of Welding. Ed. Chapman \& Hall.

Lyonnet P. (1989). Los métodos de la calidad total. Ed. Díaz de Santos, SA, Madrid.

Menon H.G. (1992). TQM in New Product Manufacturing. Ed. Mc Graw-Hill, EUA.

Montgomery D.C. (1997). Design and Analysis of Experiments. Ed. Wiley \& Sons, EUA. 


\section{Semblanza de los autores}

Sara Mercedes Cerrud-Sánchez. Es ingeniera mecánica electricista (industrial) y maestra en ingeniería mecánica por la Facultad de Ingeniería de la UNAM. Ha participado en más de 25 estudios, proyectos y asesorías para el sector industrial. Fungió como corresponsable en el proyecto Sistema experto para el análisis de falla de piezas mecánicas, metálicas. Tiene más de 25 publicaciones y actualmente es profesora del área de manufactura y materiales.

Armando Ortiz-Prado. Es ingeniero mecánico electricista y maestro en ingeniería mecánica por la Facultad de Ingeniería, UNAM. En 1989, obtuvo la distinción Universidad Nacional para jóvenes académicos en el área de docencia en ciencias exactas. Ha dirigido más de 70 tesis entre licenciatura y posgrado, así como más de 50 estudios y proyectos para el sector industrial en el área de manufactura, materiales y, en particular, análisis de falla. Tiene más de 40 publicaciones relacionadas con el área de su especialización en foros nacionales e internacionales. Actualmente es profesor y coordinador de la Unidad de Investigación y Asistencia Técnica en Materiales.

Rafael Schouwenaars. Maestro en ingeniería de minas por la Universidad de Lovaina, Bélgica. Durante cuatro años estuvo activo como investigador en varios proyectos relacionados con el estudio de la textura cristalográfica en aceros a bajo carbono en el Departamento de Metalurgia e Ingeniería de Materiales de la misma universidad. Llegó a México como experto en microscopía electrónica de barrido en el marco de una colaboración entre la Universidad de Gante y la UNAM. Desde hace dos años es profesor en la UNAM y se dedica al estudio de problemas metalúrgicos en la industria metal-mecánica mexicana, especializándose en análisis de falla y el comportamiento mecánico de materiales.

Victor Hugo Jacobo-Armendáriz. Ingeniero mecánico electricista (industrial) y maestro en ingeniería en el área de investigación de operaciones por parte de la Facultad de Ingeniería, UNAM. Ha participado en proyectos para la industria y es profesor en el área de manufactura y materiales. Actualmente trabaja en un proyecto de investigación doctoral con líneas orientadas al desarrollo de sistemas expertos en análisis de falla. 